\title{
CERTAIN CURVATURE CONDITIONS ON AN LP-SASAKIAN MANIFOLD WITH A COEFFICIENT $\alpha$
}

\author{
Uday Chand De and Kadri Arslan
}

\begin{abstract}
The object of the present paper is to study certain curvature restriction on an LP-Sasakian manifold with a coefficient $\alpha$. Among others it is shown that if an LP-Sasakian manifold with a coefficient $\alpha$ is a manifold of constant curvature, then the manifold is the product manifold. Also it is proved that a 3-dimensional Ricci semisymmetric LP-Sasakian manifold with a constant coefficient $\alpha$ is a spaceform.
\end{abstract}

\section{Introduction}

In 1989, Matsumoto [6] introduced the notion of LP-Sasakian manifolds. Then Mihai and Rosca [7] introduced the same notion independently and they obtained several results in this manifold. In a recent paper, De, Shaikh, and Sengupta [3] introduced the notion of LP-Sasakian manifolds with a coefficient $\alpha$ which generalizes the notion of LP-Sasakian manifolds. Recently, T. Ikawa and his coauthors [4], [5] studied Sasakian manifolds with Lorentzian metric and obtained several results in this manifold. The object of the present paper is to study certain curvature restriction on an LP-Sasakian manifold with a coefficient $\alpha$. After preliminaries, in Section 3 it is shown that if an LP-Sasakian manifold $M^{n}$ with a coefficient $\alpha$ is of constant curvature, then the vector field $\xi$ is a concircular vector field and as an important consequence of this theorem we prove that such a manifold is the product manifold. In the last section we study a 3-dimensional LP-Sasakian manifold with a constant coefficient $\alpha$.

\section{Preliminaries}

Let $M^{n}$ be an $n$-dimensional differentiable manifold endowed with a $(1,1)$ tensor field $\phi$, a contravariant vector field $\xi$, a covariant vector field $\eta$ and a Lorentzian metric $g$ of type $(0,2)$ such that for each point $p \in M$, the tensor $g_{p}: T_{p} M \times T_{p} M \rightarrow R$ is a non-degenerate inner product of signature

Received December 2, 2007.

2000 Mathematics Subject Classification. 53C15, 53C40.

Key words and phrases. Lorentzian Para-Sasakian manifold with a coefficient $\alpha$, manifold of constant curvature. 
$(-,+,+, \ldots,+)$, where $T_{p} M$ denotes the tangent vector space of $M$ at $p$ and $R$ is the real number space, which satisfies

$$
\begin{gathered}
\eta(\xi)=-1, \quad \phi^{2} X=X+\eta(X) \xi \\
g(X, \xi)=\eta(X), \quad g(\phi X, \phi Y)=g(X, Y)+\eta(X) \eta(Y)
\end{gathered}
$$

for all vectors fields $X$ and $Y$. Then such a structure $(\phi, \xi, \eta, g)$ is termed as Lorentzian almost paracontact structure and the manifold $M^{n}$ with the structure $(\phi, \xi, \eta, g)$ is called Lorentzian almost paracontact manifold [6]. In a Lorentzian almost paracontact manifold $M^{n}$, the following relations hold good [6]:

$$
\begin{gathered}
\phi \xi=0, \quad \eta(\phi X)=0, \\
\Omega(X, Y)=\Omega(Y, X), \text { where } \Omega=g(X, \phi Y) .
\end{gathered}
$$

In the Lorentzian almost paracontact manifold $M^{n}$, if the relations

$$
\begin{aligned}
&\left(\nabla_{Z} \Omega\right)(X, Y)= \alpha[\{g(X, Z)+\eta(X) \eta(Z)\} \eta(Y) \\
&+\{g(Y, Z)+\eta(Y) \eta(Z)\} \eta(X)], \quad(\alpha \neq 0) \\
& \Omega(X, Y)=\frac{1}{\alpha}\left(\nabla_{X} \eta\right)(Y),
\end{aligned}
$$

hold where $\nabla$ denotes the operator of covariant differentiation with respect to the Lorentzian metric $g$, then $M^{n}$ is called an LP-Sasakian manifold with a coefficient $\alpha$ [3]. An LP-Sasakian manifold with a coefficient 1 is an LPSasakian manifold [6]. If a vector field $V$ satisfies the equation of the following form:

$$
\nabla_{X} V=\beta X+T(X) V,
$$

where $\beta$ is a non-zero scalar function and $T$ is a non-zero 1 -form, then $V$ is called a torse-forming vector field [9]. In a Lorentzian manifold $M^{n}$, if we assume that $\xi$ is a unit torse-forming vector field, then we have the equation:

$$
\left(\nabla_{X} \eta\right)(Y)=\alpha[g(X, Y)+\eta(X) \eta(Y)],
$$

where $\alpha$ is a non-zero scalar function. Hence the manifold admitting a unit torse-forming vector field satisfying (2.7) is an LP-Sasakian manifold with a coefficient $\alpha$. Especially, if $\eta$ satisfies

$$
\left(\nabla_{X} \eta\right)(Y)=\epsilon[g(X, Y)+\eta(X) \eta(Y)], \quad \epsilon^{2}=1,
$$

then $M^{n}$ is called an LSP-Sasakian manifold [6]. In particular, if $\alpha$ satisfies (2.7) and the equation of the form:

$$
\alpha(X)=p \eta(X), \quad \alpha(X)=\nabla_{X} \alpha,
$$

where $p$ is a scalar function. Then $\xi$ is called a concircular vector field. A Riemannian manifold satisfying the condition $\nabla S=0$, where $S$ denotes the Ricci tensor is called Ricci-symmetric. A Riemannian manifold satisfying the condition $R(X, Y) . S=0$ is called Ricci-symmetric [8] where $R(X, Y)$ denotes 
the derivation of the tensor algebra at each point of the tangent space. Let us consider an LP-Sasakian manifold $M^{n}(\phi, \xi, \eta, g)$ with a coefficient $\alpha$. Then we have the following relations [3]:

$$
\begin{aligned}
\eta(R(X, Y) Z)= & -\alpha(X) \Omega(Y, Z)+\alpha(Y) \Omega(X, Z) \\
& +\alpha^{2}\{g(Y, Z) \eta(X)-g(X, Z) \eta(Y)\}, \\
S(X, \xi)=- & \psi \alpha(X)+(n-1) \alpha^{2} \eta(X)+\alpha(\phi X),
\end{aligned}
$$

where $R, S$ denote respectively the curvature tensor and the Ricci tensor of the manifold and $\psi=\operatorname{Trace}(\phi)$. We state the following results which will be needed in latter sections.

Lemma 2.1 ([3]). In an LP-Sasakian manifold $M^{n}$ with a non-constant coefficient $\alpha$, one of the following cases occurs:

(i) $\psi^{2}=(n-1)^{2}$.

(ii) $\alpha(Y)=-p \eta(Y)$, where $p=\alpha(\xi)$.

Lemma $2.2([3])$. In a Lorentzian almost paracontact manifold $M^{n}$ with structure $(\phi, \xi, \eta, g)$ satisfying $\Omega(X, Y)=\frac{1}{\alpha}\left(\nabla_{X} \eta\right)(Y)$, where $\alpha$ is a non-zero scalar function, the vector field $\xi$ is torse-forming if and only if the relation $\psi^{2}=(n-1)^{2}$ holds good.

\section{LP-Sasakian manifolds with a coefficient $\alpha$ which is of constant curvature}

We consider an LP-Sasakian manifold which is of constant curvature. Then we have

$$
R(X, Y, Z, W)=\frac{r}{n(n-1)}[g(Y, Z) g(X, W)-g(X, Z) g(Y, W)] .
$$

From (3.1) we have

$$
S(Y, Z)=\frac{r}{n} g(Y, Z)
$$

which implies that the manifold is Einstein and hence the scalar curvature $r$ of the manifold is given by [3]

$$
r=n\left\{p \psi+(n-1) \alpha^{2}\right\} .
$$

Putting $Z=\xi$ in (3.2) we have by virtue of (2.11)

$$
\alpha(\phi Y)=\psi \alpha(Y)+\left\{\frac{r}{n}-(n-1) \alpha^{2}\right\} \eta(Y) .
$$

Again from (3.1) we have by virtue of (2.2)

$$
\sum_{i=1}^{n} \epsilon_{i} R\left(e_{i}, Z, \phi Y, \phi e_{i}\right)=\frac{r}{n(n-1)}[\psi \Omega(Y, Z)-g(Y, Z)-\eta(Y) \eta(Z)],
$$


where $\left\{e_{i}\right\}$ is an orthonormal basis of the tangent space at any point of the manifold and $\epsilon_{i}=g\left(e_{i}, e_{i}\right)$. Now in an LP-Sasakian manifold with a coefficient $\alpha$ we have the following relation [3]:

$$
\begin{aligned}
& S(Y, Z)-\sum_{i=1}^{n} \epsilon_{i} R\left(e_{i}, Z, \phi Y, \phi e_{i}\right) \\
= & \left\{\psi \alpha(Z)-\alpha(\phi Z)-(2 n-3) \alpha^{2} \eta(Z)\right\} \eta(Y) \\
& -(n-2) \alpha^{2} g(Y, Z)+\left(p+\psi \alpha^{2}\right) \Omega(Y, Z) .
\end{aligned}
$$

Using (3.2), (3.3), (3.4) and (3.5) in (3.6) we obtain

$$
\begin{array}{r}
\left\{2(n-1) \alpha^{2}+\frac{n p \psi}{n-1}\right\} g(Y, Z)-\left\{2 \psi \alpha^{2}+\left(1+\frac{\psi^{2}}{n-1}\right) p\right\} \Omega(Y, Z) \\
+\left\{2(n-1) \alpha^{2}+\frac{n p \psi}{n-1}\right\} \eta(Y) \eta(Z)=0 .
\end{array}
$$

We consider the case when $\alpha$ is not constant. In this case, taking a frame field and contracting over $Y$ and $Z$ we obtain from (3.7) that

$$
\left[(n-1)^{2}-\psi^{2}\right]\left\{2 \alpha^{2}+\frac{p \psi}{n-1}\right\}=0 .
$$

From this equation we find either

$$
\psi^{2}=(n-1)^{2},
$$

or

$$
p \psi=-2(n-1) \alpha^{2} .
$$

If (3.9) holds, then from (3.3) we obtain

$$
r=-n(n-1) \alpha^{2},
$$

from which we find that $\alpha$ is constant, since $r$ is constant, which contradicts our assumption that $\alpha$ is non constant.

On the other hand, from (3.8) by virtue of Lemma 2.2 we conclude that $\xi$ is torse-forming. We have that

$$
\left(\nabla_{X} \eta\right)(Y)=\beta\{g(X, Y)+\eta(X) \eta(Y)\}
$$

Then from (2.6) we get

$$
\begin{aligned}
\Omega(X, Y) & =\frac{\beta}{\alpha}\{g(X, Y)+\eta(X) \eta(Y)\} \\
& =g\left(\frac{\beta}{\alpha}(X+\eta(X) \xi), Y\right) \\
\text { and } \quad \Omega(X, Y) & =g(\phi X, Y) .
\end{aligned}
$$

Now from (3.4) and using $\phi(X)=X+\eta(X) \xi$ we obtain

$$
\alpha(Y+\eta(Y) \xi)=\psi \alpha(Y)+\left\{\frac{n\left(p \psi+(n-1) \alpha^{2}\right)}{n}-(n-1) \alpha^{2}\right\} \eta(Y)
$$




$$
\begin{array}{rlrl}
\text { or, } & \alpha(Y)+p \eta(Y) & =\psi \alpha(Y)+p \psi \eta(Y) \\
\text { or } & \alpha(Y)-\psi \alpha(Y) & =p \psi \eta(Y)-p \eta(Y) \\
& \text { or, } \quad & (1-\psi) \alpha(Y) & =p(-1+\psi) \eta(Y) \\
& \text { or, } & \alpha(Y) & =p\left(\frac{-1+\psi}{1-\psi}\right) \eta(Y)=-p \eta(Y) .
\end{array}
$$

In a similar way using $\phi(X)=-X+\eta(X) \xi$ in (3.4) we obtain $\alpha(X)=-p \eta(Y)$. Since $g$ is non-singular, we have

$$
\phi(X)=\frac{\beta}{\alpha}(X+\eta(X) \xi)
$$

and

$$
\phi^{2}(X)=\left(\frac{\beta}{\alpha}\right)^{2}(X+\eta(X) \xi) .
$$

It follows from (2.1) that $\left(\frac{\beta}{\alpha}\right)^{2}=1$ and hence, $\alpha= \pm \beta$. Thus we have

$$
\phi(X)= \pm(X+\eta(X) \xi \text {. }
$$

Thus in both the cases we obtain

$$
\alpha(Y)=-p \eta(Y) .
$$

Hence we can state the following:

Theorem 3.1. If an LP-Sasakian manifold $M^{n}$ with a coefficient $\alpha$ is a manifold of constant curvature, then the vector field $\xi$ is a concircular vector field.

Again since $\xi$ is a concircular vector field, we have

$$
\nabla_{X} \xi=\alpha[X+\eta(X) \xi]
$$

where $\alpha(Y)=p \eta(Y)$, where $p$ is a scalar function.

Let $\xi^{\perp}$ denote the $(n-1)$-dimensional distribution in an LP-Sasakian manifold with coefficient $\alpha$ orthogonal to $\xi$. If $X$ and $Y$ belong to $\xi^{\perp}$, where $Y \neq \lambda X$, then

$$
g(X, \xi)=0
$$

and

$$
g(Y, \xi)=0 .
$$

Since $\left(\nabla_{X} g\right)(Y, \xi)=0$, it follows from (3.11) and (3.13) that

$$
g\left(\nabla_{X} Y, \xi\right)=g\left(\nabla_{X} \xi, Y\right)=\alpha g(X, Y) .
$$

Similarly, we get

$$
g\left(\nabla_{Y} X, \xi\right)=g\left(\nabla_{Y} \xi, X\right)=\alpha g(X, Y) .
$$

Hence

$$
g\left(\nabla_{X} Y, \xi\right)=g\left(\nabla_{Y} X, \xi\right)
$$


Now $[X, Y]=\nabla_{X} Y-\nabla_{Y} X$. Therefore

$$
g([X, Y], \xi)=g\left(\nabla_{X} Y-\nabla_{Y} X, \xi\right)=0 \text { by }(3.14) .
$$

Hence $[X, Y]$ is orthogonal to $\xi$, i.e., $[X, Y]$ belong to $\xi^{\perp}$. Thus the distribution $\xi^{\perp}$ is involutive [2]. Hence from Frobenius' theorem [2] it follows that $\xi^{\perp}$ is integrable. This implies that if an LP-Sasakian manifold with a coefficient $\alpha$ is a manifold of constant curvature, then it is a product manifold. We can therefore state the following theorem.

Theorem 3.2. If an LP-Sasakian manifold with a coefficient $\alpha$ is a manifold of constant curvature, then the manifold is the product manifold.

\section{3-dimensional LP-Sasakian manifold with a constant coefficient $\alpha$}

Let us consider a 3-dimensional LP-Sasakian manifold with a constant coefficient $\alpha$. In a 3-dimensional Riemannian manifold we have

$$
\begin{aligned}
R(X, Y) Z= & g(Y, Z) Q X-g(X, Z) Q Y+S(Y, Z) X-S(X, Z) Y \\
& -\frac{r}{2}[g(Y, Z) X-g(X, Z) Y],
\end{aligned}
$$

where $Q$ is the Ricci operator, i.e., $g(Q X, Y)=S(X, Y)$ and $r$ is the scalar curvature of the manifold.

Since $\alpha$ is constant and dimension of the manifold is 3, equations (2.10) and (2.11) reduce to

$$
\begin{gathered}
\eta(R(X, Y) Z)=\alpha^{2}[g(Y, Z) \eta(X)-g(X, Z) \eta(Y)] \\
S(X, \xi)=2 \alpha^{2} \eta(X) .
\end{gathered}
$$

From (4.2) we get

$$
R(X, Y) \xi=\alpha^{2}[\eta(Y) X-\eta(X) Y] .
$$

Putting $Z=\xi$ in (4.1) and using (4.4) we have

$$
\eta(Y) Q X-\eta(X) Q Y=\left(\frac{r}{2}-\alpha^{2}\right)[\eta(Y) X-\eta(X) Y] .
$$

Putting $Y=\xi$ in (4.5) and using (2.1) and (4.3), we get

$$
Q X=\frac{1}{2}\left\{\left(r-2 \alpha^{2}\right) X+\left(r-6 \alpha^{2}\right) \eta(X) \xi\right\}
$$

i.e.,

$$
S(X, Y)=\frac{1}{2}\left\{\left(r-2 \alpha^{2}\right) g(X, Y)+\left(r-6 \alpha^{2}\right) \eta(X) \eta(Y)\right\} .
$$

An LP-Sasakian manifold is said to be a space form if the manifold is a space of constant curvature. We assume that $\psi=$ trace of $\phi \neq 0$, i.e., $\xi$ is not harmonic [1]. 
Using (4.6) in (4.1), we get

$$
\begin{aligned}
R(X, Y) Z= & \frac{r-4 \alpha^{2}}{2}[g(Y, Z) X-g(X, Z) Y]+\frac{r-6 \alpha^{2}}{2}[g(Y, Z) \eta(X) \xi \\
& -g(X, Z) \eta(Y) \xi+\eta(Y) \eta(Z) X-\eta(X) \eta(Z) Y]
\end{aligned}
$$

Hence we can state the following:

Theorem 4.1. A 3-dimensional LP-Sasakian manifold with a constant coefficient $\alpha$ is a space form if and only if the scalar curvature $r=6 \alpha^{2}$.

Next we consider a 3-dimensional LP-Sasakian manifold with constant coefficient $\alpha$ which satisfies the condition

$$
R(X, Y) . S=0 .
$$

From (4.8) we have

$$
S(R(X, Y) U, V)+S(U, R(X, Y) V)=0 .
$$

Again from (4.2) we get

$$
R(X, \xi) Z=\alpha^{2}[\eta(Z) X-g(X, Z) \xi] .
$$

Putting $Y=\xi$ in (4.9) and using (4.10) we get

(4.11) $\eta(U) S(X, V)-g(X, U) S(\xi, V)+\eta(V) S(U, X)-g(X, V) S(U, \xi)=0$.

Since $\alpha^{2} \neq 0$ using (4.3) in (4.11) we have

(4.12) $\eta(U) S(X, V)-2 \alpha^{2} g(X, U) \eta(V)+\eta(V) S(U, X)-2 \alpha^{2} g(X, V) \eta(V)=0$.

Taking a frame field and contracting over $X$ and $U$ from (4.12) we obtain

$$
S(\xi, V)-8 \alpha^{2} \eta(V)+r \eta(V)=0 .
$$

Using (4.3) in (4.13) we obtain

$$
\left(r-6 \alpha^{2}\right) \eta(V)=0 .
$$

This gives $r=6 \alpha^{2}$ (since $\eta(V) \neq 0$ ), which implies by Theorem 4.1 that the manifold is a space form.

Hence we can state the following:

Theorem 4.2. A 3-dimensional Ricci semi-symmetric LP-Sasakian manifold with a constant coefficient $\alpha$ is a space form.

Since $\nabla S=0$ implies $R(X, Y) . S=0$, we get the following:

Corollary. A 3-dimensional Ricci symmetric LP-Sasakian manifold with a constant coefficient $\alpha$ is a space form. 


\section{References}

[1] T. Adati and A. Kandatu, On hypersurfaces of P-Sasakian manifolds and manifolds admitting a concircular vector field, Tensor (N.S.) 34 (1980), no. 1, 97-102.

[2] F. Brickell and R. S. Clark, Differentiable Manifold, Van Nostrand Reinhold Comp. London 1978.

[3] U. C. De, A. A. Shaikh, and A. Sengupta, On LP-Sasakian manifolds with a coefficient $\alpha$, Kyungpook Math. J. 42 (2002), no. 1, 177-186.

[4] T. Ikawa and M. Erdogan, Sasakian manifolds with Lorentzian metric, Kyungpook Math. J. 35 (1996), no. 3, Special Issue, 517-526.

[5] T. Ikawa and J. B. Jun, On sectional curvatures of a normal contact Lorentzian manifold, Korean J. Math. Sci. 4 (1997), 27-33.

[6] K. Matsumoto, On Lorentzian paracontact manifolds, Bull. Yamagata Univ. Natur. Sci. 12 (1989), no. 2, 151-156.

[7] I. Mihai and R. Rosca, On Lorentzian P-Sasakian manifolds, Classical analysis (Kazimierz Dolny, 1991), 155-169, World Sci. Publ., River Edge, NJ, 1992.

[8] L. Verstraelen, Comments on pseudo-symmetry in the sense of Ryszard Deszcz, Geometry and topology of submanifolds, VI (Leuven, 1993/Brussels, 1993), 199-209, World Sci. Publ., River Edge, NJ, 1994.

[9] K. Yano, On the torse-forming directions in Riemannian spaces, Proc. Imp. Acad. Tokyo 20 (1944), 340-345.

Uday Chand De

Department of Mathematics

UNIVERSITY OF KALYANI

KALYANI-741235, INDIA

E-mail address: uc_de@yahoo.com

KADRI ARSLAN

Department of Mathematics

ULUDAG UNIVERSITY

16059 Bursa, Turkey

E-mail address: arslan@uludag.edu.tr 